\title{
Future developments in allergy prevention: a matter of integrating medical, natural and social sciences
}

\author{
Luud J.W.J. Gilissen ${ }^{1,2, *}$, Harry J. Wichers ${ }^{1,3}$, Huub F.J. Savelkoul ${ }^{1,4}$ and \\ George Beers ${ }^{1,5}$
}

\begin{abstract}
Allergy is a multifactorial disease and has a significant impact on society. Strategies for allergy prevention require integrated and multidisciplinary approaches. A knowledge framework has been designed for this purpose to be helpful in the identification of known and lacking scientific details, and to visualize and coordinate the various activities in the development of a particular prevention strategy. In this framework, the development of allergy prevention strategies is on the edge of medical and natural sciences on the one hand and social sciences, including economics, communication and education, on the other. First, fundamental research activities are carried out to understand the characteristics of the patient, the allergen and the way these two interact. Next, the resulting knowledge enables the design of applications for allergy prevention strategies, directed to diagnosis, detection, reduction of allergen loads and changes in life style. Such designs may aim at society as a whole but also at the individual patients, depending on the need. Then, the framework also includes the approval by and implementation into society of a designed prevention strategy. At last, education and dissemination form a necessary category in the framework to establish a solid knowledge infrastructure embedding the allergy prevention strategy. Such framework approaches for the development of prevention strategies require the development of strict cooperation in extended research consortia at national and international level. Examples of such consortia are described.
\end{abstract}

Keywords: allergy prevention strategies; consortial approach; knowledge framework

\section{Introduction}

The prevalence of IgE-mediated allergic diseases is increasing among the populations in developed countries. Nowadays, about $25 \%$ of these populations suffer from one or more forms of allergy, like asthma, hay fever, eczema and food and

\footnotetext{
${ }^{1}$ Allergy Consortium Wageningen, Wageningen University and Research Centre, P.O.Box 16, 6700 AA Wageningen, The Netherlands

${ }^{2}$ Plant Research International, Wageningen University and Research Centre, P.O.Box 16, 6700 AA Wageningen, The Netherlands

*Corresponding author: E-mail: luud.gilissen@wur.nl

3 Agrotechnology \& Food Innovations, Wageningen University and Research Centre, P.O.Box 17, 6700 AA Wageningen, The Netherlands

${ }^{4}$ Dept. of Cell Biology and Immunology, P.O.Box 338, 6700 AH Wageningen, The Netherlands

5 Agricultural Economic Research Institute (LEI), Wageningen University and Research Centre, P.O.Box 29703, 2502 LS The Hague, The Netherlands
} 
contact allergies. The prevalence of hay fever alone in young adults amounts even to $30 \%$ (Bauchau and Durham 2004). Half of these have allergic complaints and are already under medical treatment; the other half are potentially at risk due to their genetic background. This background is reflected in the family history of allergic diseases and can be analysed by determining the degree of polymorphisms of currently known loci of immune-response genes (like genes for cytokines and their receptors) involved in allergic diseases. This genetic risk can subsequently be modulated by interactions with the environment or by a specific physiological condition.

Allergy causes a high burden on the economy. Direct plus indirect annual costs have been estimated for Western Europe at about 45 billion Euros (price level 2004) (UCB 1997; 1999; Gilissen et al. in press). Hardly impossible to quantify in terms of money is the reduced 'quality of life' of allergic patients themselves, and if they are little children, also the reduced 'quality of life' of their parents, carers and family members. In addition, from May 2004 onwards, new member states from Eastern Europe have joined the European Union. Their 100 million inhabitants will soon become adapted to the westernized life style. This will certainly include a drastic rise in the allergy problem in these countries, as has been seen after the German reunion (Von Mutius 1998). These facts make time urgent to develop strategies for large-scale tackling of the allergy problem. The recently started Allergy Consortium Wageningen aims at designing strategies for the prevention, symptom reduction and management of allergy.

\section{Bases to allergy prevention}

Roughly, we can distinguish between inhalation allergies, food allergies and contact allergies, each related to a specific surface of the human body through which an allergen can come into contact with the immune system: the respiratory tract with a surface of about $80 \mathrm{~m}^{2}$, the intestine representing $300 \mathrm{~m}^{2}$, and $2 \mathrm{~m}^{2}$ of skin have to be defended against pathogens. Zooming in to specific allergens, we see that some allergens follow single routes, whereas others can act through more than one route. For example, some allergens that can cause airway allergies like hay fever (e.g. PR10 proteins) occur as homologues in some fresh fruits and vegetables and may cause food allergy (oral allergy syndrome). Some skin contact allergens (e.g. latex proteins) can cause food allergy due to cross-reactivity with IgEs in the digestive tract. Other allergens are pan-allergens, occurring in many (food) organisms. These types of allergy are so-called IgE (Immunoglobulin type E)-mediated allergies, which are different from cell-mediated allergies, prominently typified by coeliac disease (CD) or gluten intolerance.

Allergy is a complex problem and a multifactor disease. Allergy is a collective noun for aberrant and violent reactions of the immune system against harmless compounds, normally proteins, which should be tolerated by the immune system. Three factors are generally relevant in the development of allergies: (1) the genetic constitution of the (potential) patient; (2) the presence of allergenic compounds (in the air, in food, by contact); and (3) adjuvant factors from the physicochemical, the (micro)biological and the social environment. Allergies develop from continuous interactions of these factors with the immune system. The etiology distinguishes two phases. These are: (1) the phase of allergic sensitization without symptoms, during which the immune system is primed, and (2) the phase of specific clinical responses (symptoms) occurring due to repeated contacts with the allergenic compounds. 
At the level of cell types of the immune system, Th1, Th2 and regulatory $\mathrm{T}$ cells (Treg cells) are involved in the development of inflammatory diseases. Especially, Treg cells produce suppressory cytokines and are part of an anti-inflammatory network that keeps inflammatory $\mathrm{T}$ cells (both $\mathrm{Th} 1$ and $\mathrm{Th} 2$ ) and their downstream effectors under control. Absence of Treg cells results in unbalanced expression of Th1 and Th2 cells. Allergy results from a skewed balance towards Th2 cells. When Th1 cells predominate, auto-immune diseases like diabetes type 1 may develop. It is suggested that a high burden by pathogens during early childhood plays a crucial role in the education of the immune system towards the development of a strong regulatory network with the presence of Treg cells preventing Th1- and Th2-related diseases. Treg cells are especially crucial in the phase of sensitization (Yazdanbakhsh, Kremsner and Van Ree 2002; Robinson, Larche and Durham 2004).

Some types of allergy, and in particular contact allergy, can be classified as a delayed hypersensitivity response. This is because the reaction is not due to antibodies but to the activity of lymphoid cells. Such reaction usually occurs within 24 to 48 hours after contact with the allergen. In this case, the allergen crosses the skin barrier and will be presented to $\mathrm{T}$ cells by skin dendritic cells in the draining lymph nodes. After a first wave of release of chemotactic mediators, inflammatory macrophages, granulocytes and $\mathrm{T}$ lymphocytes are attracted to the skin site. By subsequent cytokine release, lymphocytes like Th1 cells, CD8+ cytotoxic cells and monocytes produce a local inflammatory response while clearing the allergen (Saint-Mezard et al. 2004). In the case of coeliac disease, gluten is partially degraded and modified by tissue transglutaminase. The resulting peptides bind to HLA-DQ2 or HLA-DQ8 receptors and trigger $\mathrm{T}$ cells to an inflammatory response causing destruction of epithelial cells of the small intestine (Sollid 2002).

The medical approach is in general a straightforward strategy from diagnosis to the treatment of allergy symptoms. There is, however, a steadily growing awareness that prevention strategies can be developed which precede and connect to the medical phase. These strategies should focus (1) on the allergen in reducing the allergenic load to (potential) patients in environment and food; (2) on life-style factors and how these should be changed; and (3) on food components and/or intestinal micro-organisms that are able to modulate the immune system towards a state of tolerance (i.e. a balanced T-cell state).

Such premedical strategies thus include more complex, integrated and often indirect approaches, requiring the acquisition of knowledge from different scientific disciplines and the implementation of this knowledge and its practical results into society. Increasingly, this approach is also appreciated to be more cost-effective for society and provides new business leads to the pharmaceutical industry. Whereas medical treatments are especially directed to the individual patient, the premedical prevention strategies focus on groups of patients and on society as a whole. Here, the contours become visible of a knowledge framework for an integrated approach of allergy prevention.

\section{Knowledge framework}

The Allergy Consortium Wageningen proposes a knowledge framework for the development of premedical allergy prevention strategies. Such framework runs from fundamental research to societal implementation and education. Fundamental research is required for better understanding of the phenomenon with regard to etiology and allergenicity. Designing research should use the fundamental knowledge for technical 
development of strategies for better management of the allergy problems. In a next step, these strategies should become implemented in society. Without acceptance by society, these strategies will not be viable. And at last, a solid infrastructure through dissemination and education should be established in society to reach continuity and sustainability of the strategies, or more in general, to make society familiar with this multidisciplinary and integrated approach.

1. Fundamental research.

The starting point is comprehensive research, including the fundamental genetic and immunological mechanisms that make a person predisposed to become or to be allergic. What kinds of genetic and physiological markers characterize such individual? How do environmental and life-style factors add to or prevent against the development of allergy, in individual cases or in entire populations? What is the effect of the composition of the intestinal micro-flora? On the other hand, fundamental characteristics can be ascribed to proteins that make them allergenic. These characteristics relate to their primary, secondary and tertiary structures, their linear and conformational epitopes, and their exposition to the immune system (Jenkins et al. 2005). Medical sciences and human genomics, plant and animal sciences and their functional genomics and proteomics, environmental sciences including aspects of biodiversity, etc. are leading in this context.

2. Designing research.

The next category in the framework of knowledge relates to designing research. Here, data from fundamental research are becoming strategically applied. This step includes the designing of tools for diagnosis, e.g. to detect and quantify allergens in food products and in the indoors and outdoors environment. Also, tools can be developed for the diagnosis of (potential) patients, and methods can be designed to make food products hypoallergenic, e.g. through the modification of crops by selection, breeding or molecular techniques, or through processing technologies. In addition, methods can be developed directed towards behavioural changes of consumers aiming at avoidance of allergens or better coping with the disease. But also the restructuring of the green living environment is a relevant issue in designing research. These designs need to be tested in real-life situations to become an essential part in a prevention strategy.

3. Implementation phase.

This category relates to implementation of the strategy into society. At this stage, a great variety of stakeholders become involved. For example, the development of an allergen-free food production chain involves primary producers, food producers, retail, the general and the allergic consumers, (inter)national governmental bodies, etc. It relates to information supply to and between stakeholders. Such implementations can result at the small-scale level in dietary advices with regard to specific allergies (e.g. peanut allergy), the development of consumer-friendly databases for scanning food products in supermarkets, and as large-scale implementations in society through the building of allergen-free city quarters, etc.

4. Dissemination of knowledge.

Circulation of the knowledge acquired in the former categories of the framework should be used for the establishment in time of a solid infrastructure. This needs the development of platforms for information and education at different levels that enable the dynamic and interactive dissemination of knowledge, skills and experience in society. At universities, this can be elaborated in regular allergy courses and in post-graduate and $\mathrm{PhD}$ programmes. A specifically useful tool for dissemination of knowledge is the Internet (e.g. www.allergymatters.org). 
Table 1. Knowledge framework in allergy prevention

\begin{tabular}{|l|l|l|l|l|l|}
\hline \multicolumn{1}{|c|}{ Activity } & $\begin{array}{l}\text { Comprehensive } \\
\text { research }\end{array}$ & $\begin{array}{l}\text { Designing } \\
\text { research }\end{array}$ & $\begin{array}{l}\text { Prevention } \\
\text { strategies }\end{array}$ & $\begin{array}{l}\text { Implementation } \\
\text { in society }\end{array}$ & $\begin{array}{l}\text { Knowledge } \\
\text { circulation and } \\
\text { education }\end{array}$ \\
\hline Inhalation & & & & & \\
\hline Food type & & & & & \\
\hline $\mathrm{CD}^{\mathrm{a}}$ & & & & \\
\hline Contact & & & \\
\hline
\end{tabular}

The four categories of activities in the knowledge framework are visualized in the matrix of Table 1. This matrix can be fine-tuned further to specific items within an allergy type, or directed towards a prevention strategy for a specific allergy. The matrix is helpful in the identification of known and lacking scientific details and unanswered research questions, concerning a specific allergy as well as its causing allergen. It enables to visualize and coordinate the various activities into a coherent framework of knowledge. The matrix shows that allergy prevention is on the edge of natural/medical sciences and social sciences and makes the multidisciplinarity clear. To achieve multidisciplinarity, the cooperation and integration of research activities from the various disciplines into (larger) consortia is a prerequisite. Below, examples of such consortia are described.

\section{Research consortia}

Several national and international consortia develop integrated and multidisciplinary strategies focusing on the generation of knowledge to tackle specific allergy problems from the molecular to the societal level. Examples of such consortia are given below.

PROTALL. Internationally, in order to study and discuss the complex problems of food allergy, an EU-funded network (PROTALL) (FAIR CT 4356) has been set up in 1999 , bringing together a wide range of clinicians and natural and social scientists. In all, 35 partners have been involved. The aim of this network was the identification of biological and molecular characteristics that make a protein allergenic, and to further inventory allergenic (food) sources.

EUROPREVALL. Recently, a follow-up of the PROTALL network has been established in the EU Integrated Project EUROPREVALL, which is under negotiation now by the EC. This IP includes 54 partners from all over Europe. It basically aims at investigating the true magnitude of the food-allergy problem via performing food challenges, and by improving diagnostic methods. Main attention is on the assessment of the prevalence of food allergies in adults and children, the patterns of allergenic foods across the major climatic and cultural regions of Europe, and the impact of environmental and socioeconomic factors. Next to this epidemiological approach, 
novel diagnostic and predictive tools and methods to enable more effective management of the food-allergy problem will be developed. This information will become useful in the implementation of risk management measures, including the detection of allergens in food products.

$E U-S A F E$. Another international activity is the EU-SAFE (QLK1-CT-2000-01394) project aiming at a 'field-to-table' approach and covering the entire chain of knowledge from fundamental medical and plant genomics research to the participation of the patient society. In this project, partners are involved from hospitals and medical science institutes, from agriculture and food research institutes, from plant-breeding companies, fruit-juice industry and from the European Asthma and Allergy Association (EFA). Some of the results of this project (specifically those related to the production of hypoallergenic fruit) are elaborated in a separate chapter of this book.

The Allergy Consortium Wageningen (ACW). In The Netherlands, the Allergy Consortium Wageningen (ACW) is an expertise centre on allergy prevention funded by Wageningen University and Research Centre. Wageningen UR consists of five Science Groups, related to plant, animal, food, environmental and social sciences, respectively. The ACW includes representatives of all five Science Groups, and works among other things on the birch-fruit-vegetable allergy syndrome. About $10 \%$ of the Northwest-European population suffers from hay fever due to sensitization by birch pollen. This allergy may proceed further in patients to the development of food allergy against fresh fruits, vegetables and tree nuts as a result of IgE cross-reactivity to Bet $\mathrm{v} 1$ homologues in these foods. Bet $\mathrm{v} 1$ is the major allergen of birch. Using molecular and genomics techniques, the focus is directed to the biological diversity in allergenicity of different birch species in order to identify hypoallergenic birch genotypes for planting in the urban environment, or to produce hypoallergenic birch trees through breeding or molecular techniques. The human attitude towards plantations of GM trees with non-allergenic characteristics is under investigation. The results of this study will lead to advising national and local authorities on urban design and decoration of the green environment. At the level of Bet v 1-related crossreactive food allergens, research is carried out on the species-specific differences (e.g. between apple, carrot and celery) in allergen stability during food processing. Also, consumption patterns are studied in relation to the development of food allergies. Here, attention is given to the basic immunopathological changes underlying allergic sensitization. Another research activity of the ACW relates to allergy with regard to GM and non-GM novel foods. Furthermore, research is performed, together with industrial partners, on allergies to nuts and seeds.

Coeliac disease - The Coeliac Disease Consortium (CDC). Another Dutch example is the Coeliac Disease Consortium (CDC), funded by the Dutch Government (BSIK03009), aiming at safer foods and effective diagnosis, prevention and therapy of coeliac disease. Partners in this consortium are Leiden University Medical Centre (LUMC), University Medical Centre Utrecht (UMCU), TNO Food Research, Wageningen University and Research Centre, the industrial partners Cebeco Seeds, DSM Food Specialties and Association des Amidonneries de Céréales de l'Union Européenne (AAC), and the Netherlands Coeliac Disease Society. Coeliac disease is a chronic intestinal disorder affecting up to $1 \%$ of the western population. About $85 \%$ of the patients are not properly diagnosed. The only treatment for CD is a strict, lifelong gluten-free diet. The CDC includes a Human Genomics research cluster and a Food Genomics research cluster. The Human Genomics cluster focuses on the identification of aberrant molecular pathways leading to the disease development in 
order to improve diagnostic procedures and to design a rational screening programme for the identification of children at risk of developing CD. Further, leads are under development for novel therapies based on fundamental insight into the role of particular molecules and molecular pathways in the onset of CD. Within the Food Genomics cluster, research is concentrated on the reduction of intake of toxic gluten through improving means for the detection of toxic gluten peptides in food products and the evaluation of novel approaches for the degradation of toxic gluten peptides in food. Further, rational tools are under development to aid breeding programmes for the generation of safer wheat varieties. The Human and Food research clusters are supported by a Bioinformatics programme for data integration and software development for data analysis. In the Societal Research programme, the impact and acceptance of new foods, therapeutic, diagnostic and screening tools are under investigation. The central aim of the CDC is the improvement of the quality of life of CD patients.

\section{Conclusion}

It is clear that allergy is a growing problem that can be tackled at best through broad and multidisciplinary approaches directed towards long-term strategies geared primarily to prevention rather than cure. Such strategies relate to: (1) avoidance of contact with allergens, and (2) adequate changes in relevant life-style factors. They require integration of basic scientific research to understand the etiology of the disease, the exposition of the allergens to the patient's immune system, and the reinforcing and intensifying effects of adjuvant factors. Medical, natural and social research complement each other in this field, enabling the development and implementation of suitable multidisciplinary strategies for sustainable long-term allergy prevention that are accepted by society.

\section{References}

Bauchau, V. and Durham, S.R., 2004. Prevalence and rate of diagnosis of allergic rhinitis in Europe. European Respiratory Journal, 24 (5), 758-764.

Gilissen, L.J.W.J., Van Heteren, G., Van der Meer, I.M., et al., in press. Towards prevention of allergy through an integrated multidisciplinary approach. NJAS Wageningen Journal of Life Sciences.

Jenkins, J.A., Griffiths-Jones, S., Shewry, P.R., et al., 2005. Structural relatedness of plant food allergens with specific reference to cross-reactive allergens: an in silico analysis. Journal of Allergy and Clinical Immunology, 115 (1), 163-170.

Robinson, D.S., Larche, M. and Durham, S.R., 2004. Tregs and allergic disease. Journal of Clinical Investigation, 114 (10), 1389-1397.

Saint-Mezard, P., Berard, F., Dubois, B., et al., 2004. The role of CD4+ and CD8+ T cells in contact hypersensitivity and allergic contact dermatitis. European Journal of Dermatology, 14 (3), 131-138.

Sollid, L.M., 2002. Coeliac disease: dissecting a complex inflammatory disorder. Nature Reviews. Immunology, 2 (9), 647-655.

UCB, 1997. European allergy white paper. The UCB Institute of Allergy, Brussels. [http://www.theucbinstituteofallergy.ucb.be/WhitePaper/]

$\mathrm{UCB}, 1999$. European allergy white paper. Update. UCB Institute of Allergy, Brussels. 
Chapter 1

Von Mutius, E., 1998. The rising trends in asthma and allergic disease. Clinical and Experimental Allergy, 28 (Suppl. 5), 45-49; discussion 50-1.

Yazdanbakhsh, M., Kremsner, P.G. and Van Ree, R., 2002. Allergy, parasites, and the hygiene hypothesis. Science, 296 (5567), 490-494. 